\title{
Personalized Handling of Semantic Data with MIG
}

\author{
Mariano Ricu, David Camacho \\ Computer Science Department \\ Universidad Autónoma de Madrid, Spain \\ \{mariano.rico, david.camacho\}@uam.es
}

\author{
Óscar Corcho \\ Ontology Engineering Group \\ Departamento de Inteligencia Artificial \\ Universidad Politécnica de Madrid, Spain \\ ocorchoofi. upm.es
}

\begin{abstract}
This paper shows a semantically-enthled web application nomed MIG used to create user profiles which enhances users accessibility by allowing the creation of an user interface adapted to the user needs, the device used, and its proferences. This appmach exploits the Semantic Web technologies and the infrastructure and applications created in previous work
\end{abstract}

\section{Introduction}

According to d'Aquin et al [1], in 2007 there were around 23,000 ontologies available on the Intcrnet, and semantic data have grown exponentially for the last ten years [2], especially after the Linked Open Data initialive ${ }^{1}$. And the maturity of semantic technologies is demonstrated by its usc in a wide number of applications nowadays [3].

However, despite this wealth of information, the Semantic Web envisioned by its authors is still far away. In their envision [4], semantic agents specialized in human-computer interaction are able to act as our majordomes in the Weh. These agents should create an user interface adapted to the user needs, specifically its interaction device (e.g. PC, handheld or TV), its characteristics (e.g. color blindness or reduced visual sharpness), or its preferences (e.g acsthetic or corporative image). This may secm an unnecessary requirement, but semantic agents must be able to solve this problem becausc in the vision of the Scmantic Web, the semantic agents spccialized in interacting with humans are responsible for creating a suitable user interface.

Figure 1 shows the change in the way users and companies interact. Part (a) of this figure shows the current situation, in which users interact directly with comipanies by mears of wcb applications. Although there cxist web hubs, such as those for finding flight tickels, the most common situation is a one-on-onc relation between a user and a given company through the company web site. The Semantic Web promises semantic agents able to aggregate semantic data from different companies and offer to the users a unified user interface, as shown in part (b). The big deal for these companies is that creating a web site is not mandatory, and they have to focus only on providing semantic data.

\section{See hitp:/linkeddata.org}

The approach presented in this paper is aimed at bridging this gap by using a semantically-enabled application named MIG in conjunction with VPOET [5], as shown in part (b) of figure 1. MIG allow users create and store user profiles, with data about its interactive needs, its interaction device, and its aesthetic preferences. VPOET stores web templates created by web designers. The hypothetical semantic agent shown in part (b) of figure 1 could exploit the information provided by VPOET and MIG to create a personalized web interface.

The next section shows briefly Fortunata and VPOET. Section 3 shows how VPOET can exploit MIG profiles to provide the "most adequate"template for a given user profile. Section 4 presents related work, and section 5 presents conclusions and future work.

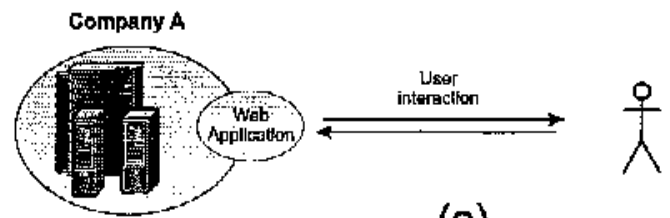

(a)

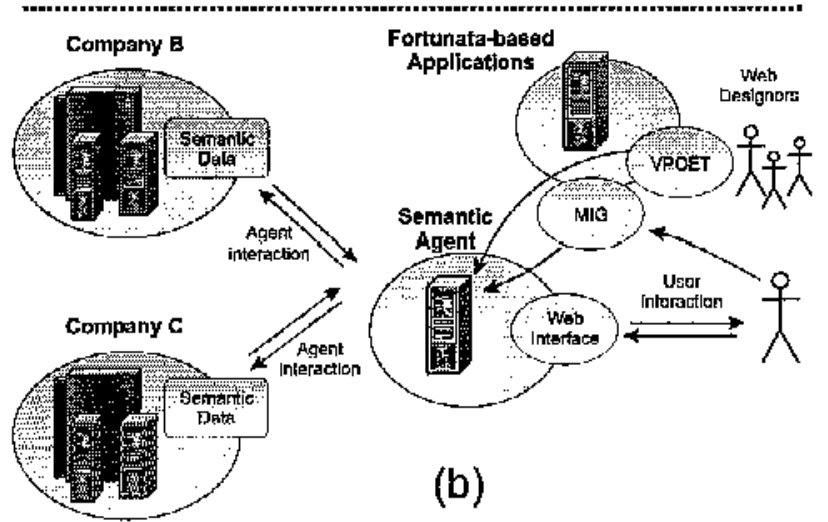

Fig. 1. Changes in the relationships between users and companies due to the Semantic Web paradigm. (a) Traditional way. (b) Sernantic Web envision and our approach.

\section{Fortunata and VPOET}

This section summarizes Fortunata [6], the underlying infrastructure used by MIG, as well as other Fortunata-bascd 
application named VPOET which exploits the semantic user profile stored by MIG.

\subsection{Fortunata to Create Semantically-Enabled Web Applications}

Fortunata ${ }^{2}$ is a Java library built on top of the JSPWiki wiki engine, whose main fealures are its support for the management of forms and its extensibility capabilities by means of plugins.

Fortunata simplifies the creation of semantically-enabled web applications by delegating to the underlying wiki engine the client-side presentation and scrver-side publication of semantic data. The creation of pages is done with a wikibased syntax, which has predefined constructs to create links, sortable tables, tables of contents, etc. The publication of semantic data is done automatically by the system, which also includes an easy-to-ttse mechanism to add links to these data from any wiki page.

A Fortunata-based application consists in a set of wiki pages that contain regular wiki code intertwited with calls to $\mathbf{F}$ plugins. For instance, VPOET is a Fortunata-based application that consists in four interrelated wiki pages and seven Fplugins.

The main assumptions that motivated our work in Fortunata wcre that we can clearly separate the roles involved in the crcation of semantically-enabled web applications and that the skills required for this can be drastically reduced if we provide adequate tools for each of these roles.

\subsection{VPOET to Handle Semantic Data in Web $\mathbf{A p}$ - plications}

VPOET is a Fortunata-based web application oriented to enable client-side web designers, also known as "template providers", to create web templates lor a set of ontology components. These templates can be used to visualize semantic data (output templates) or to request it from users (input templates). For example, let's imagine that we want to create output and input templates for the concept Person in the FOAF ontology. These templates can be used to tender any data source containing instances (individuals) of this class (or any subciass if there are no more specialized templates for them), and to present a form to request data that will be converted to an instance of Pergon, respectively.

VPOET is focused on Web designers, who should be able to author attractive designs capable of handling semantic data. Hence, VPOET only requires basic skills in client-side technologies (e.g., HTML, Javascript). The most difficult task to be performed by such devclopers is to embed some semantic data management macrus in the client-side web code (HTML, CSS, or Javascript) gencraled by the web designer favorite authoring tool (eg. Drcamweaver). Hence there is little training

2. See ht.tp//tshtar.ii.nam.es/fortunata nceded to start creating templates (a 30 min. online tutorial ${ }^{3}$ is cnough, as showed in our evaluation).

From the point of view of end users who browse through the visualization of semantic data sources gencrated by output templates or who have to introduce semantic data with input templates, a VPOET-enabled application is like any other web application, with information shown in tables or any other HTML clement, and usual HTML forms with text fields, radio buttons, clc.

VPOET has two faces, on the one hand it is a web application orienled to web designers ranging from amateur users to professional ones. On the other hand, it is a semantic data source fed by the templates created by a community of web designers sharing and reusing templates.

This source can be exploited easily by common web dcvelopers, in any programming language, by means of HTTP messages (GET and POST) like "render the semantic data at URL $\mathrm{Z}$ by using the output template $\mathrm{X}$ created by designer $\mathrm{Y}$ ", codified as a HTTP GET message by means of the following URL:

hetp: / / URt - to-servlet/vPoetReguestservlet?

action=renderoutput.\&.

designID $=\mathrm{X} \varepsilon \mathrm{d}$

provider $=Y \&+3$

sotrece $=\mathrm{z}$

Note: The symbol $\rfloor$ means that the URL has been splitted for readability.

An additional argument 1 ndvID specify a given individual in the source. In this case, only the individual is rendered. The full syntax of these macros and HTTP messages can be found in the aforementioned tutorial.

A Googlc Gadget named GG-VPOET ${ }^{4}$ cxploits VPOET templates by means of the aforementioned HTTP messages. By using this gadget, any end user can render a semantic data sourcc or provide a web interface to crealc semantic data. GGVPOET, as any other Google Gadget, can be inserted into a rcgular web page or in Google products such as iGoogle, Google Desktop or Google Pagses.

\subsection{Extending VPOET to Support Personalization}

Following the previous example, if the parameters designID and provider (that specify uniquely a temiplate) are missing, and the parameter object (e.g. FOAF . Pergon) is specified, VPOET retrieves the templates designed for that object and uses anyone of them to render the given semantic data sourcc. But if the parameter userprofile is specified, VPOET should return the most adeçuatc template for that user profilc. This parameter is a URL pointing to a MIG user profilc. The following HTTP message shows an example:

Ittp: / / URr - to-servlet/VPoetRequestservlet'] actionerenderoutpute.

gaurcenazd

3. See http:/ishtar.ji.uam.es/fortunata/wiki.jsp?page=VPOETTutorial 4. Available at the Google Gadgets Directory (Hilp:/www.gnogle.com/ig/ direclory? (Lype=gatigets) 


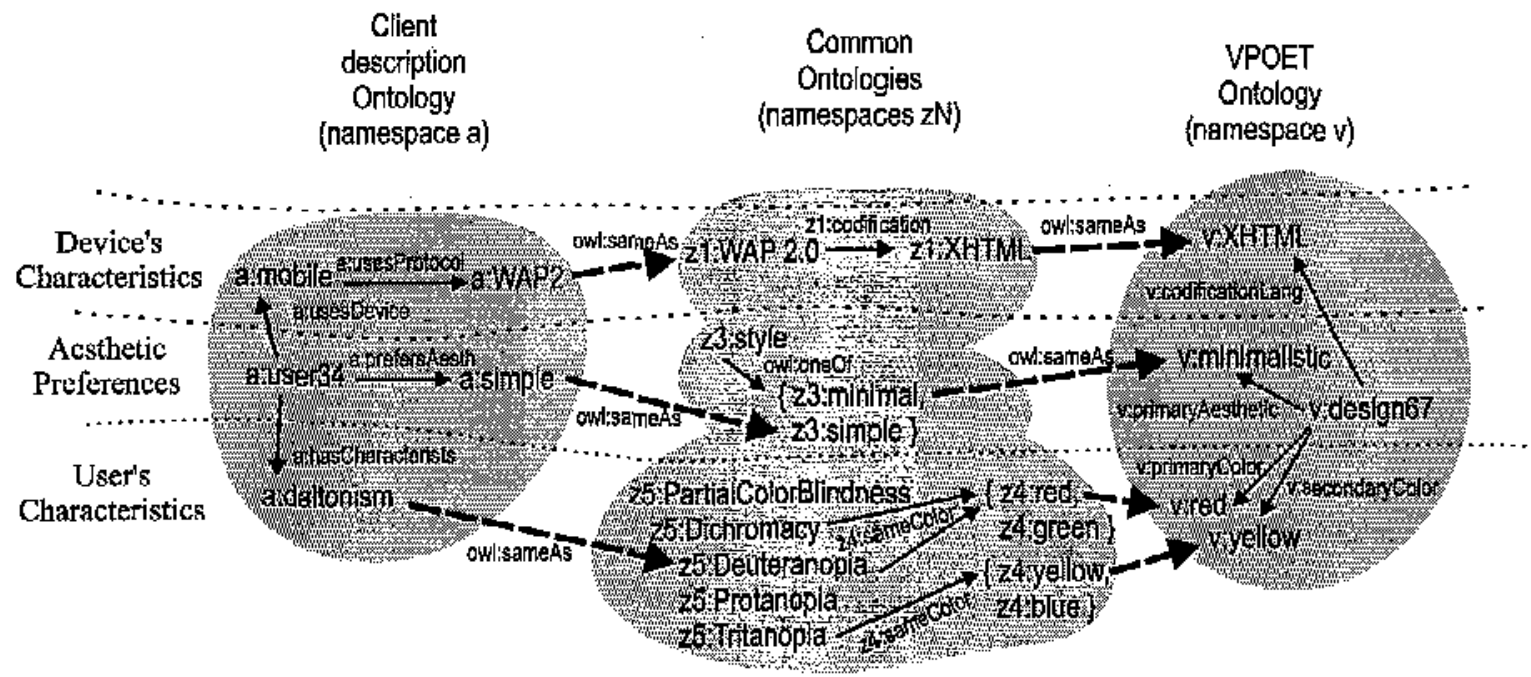

Fig. 2. Integrating semantic data from VPOET and MlG.

object=foat . Personc.

userProf 1 lemttp / / URt-to-MIG/mig, data.owl\#pIotile8g Note: The symhol \# must be codified as $\$ 23$ to be parsed correctly by VPOET.

\section{Creating a User Profile with MIG}

MIG 5 stands for "Me InteractinG". It is a Fortunatabased web application oriented to cominon users interested in specifying their profile. This profile comprise details about vision impaiment, device used and aesthetic prefercnces, as shown in figure 3. As any other Fortunata-based application, MIG stores the information provided by its users as semantic data easily reachable at a well known URL.

An example of matching VPOET tcmplates and a given MIG user profile is depicted in figure 2. Each ontology, identified by a namespace, is shown as a cloud. The elements of the ontology, and their individuals, are shown inside its cloud. The left part of this figurc shows the ontology describing the user profile, characteriscd by namespace $a$. In this example, the uscr identificd as a:user34 has the following profile: (1) uncs a WAP2 mobilc phone as interaction device, (2) prefers simplc aesthetics and (3) he/she is daltonic (colour-blindness associaled to red-green colours).

In centre part of figure 2, public well-known ontologies are shown. Ontology $z_{1}$ indicates that the protocol WAP2,0 is codified as XHTML. For ontology $z_{3}$,"minimal"and "simple"are different kinds of styles but semantically close. Ontology $z_{5}$ has a visual-impairments hierarchy.

The right part of figure 2 shows the VPOLT ontology, with namespace $v$. In this ontology, the template identified as v:design67 is codified using the XHTML language, its primary aesthetic is minimalistic, and it has red and yellow as primary and secondary colours.

\section{S. See http:/ishtar.ii.uam.es/fortunata/Wiki.jsp?page=MIG}

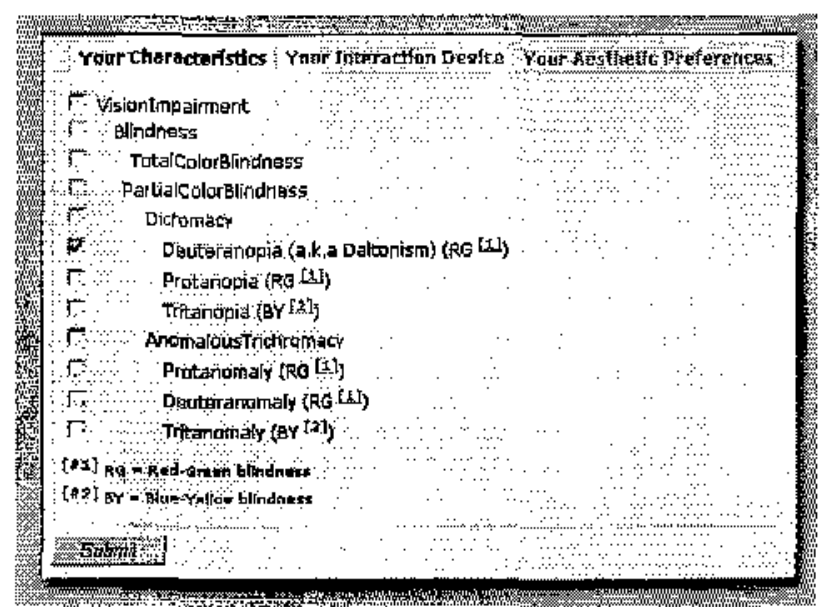

Fig. 3. User characteristics in MIG

With this semantic information is impossible to find that v:design67 is a valid template for a:user 34 . An additional scmantic data source is required in order to link elements bclonging to different ontologies. These links use to be "sameAs"(tcchnically there are three types: owl:sameAs, owl:cquivalentClass and owl:equivalentProperty to distinguish individuals, classes, and properties/relations respectively) relations, shown as discontinuous bold arrows in figure 2 . Joining all this semantic information, a semantic query (e.g., by using SPARQL language) based in the user profile, like this one: "selett a template with these characteristics: (1) codified in XTHML, (2) with minimalism as chief aesthetic, and (3) with primary colors avoiding red and green tones for text and background"would retum a personalized template. For this example, the result of this query would be the design v:design67. 


\subsection{Public Ontologies Evaluation}

The Linked Data initiative recommends reusing ontologies. Although we have used ad hoc ontologies for VPOET and MIG, we have reviewed some public ontologies and some parts have been used by MIG.

The MIG visual impairment part has been inspired by the works of Karim \& Tjoa [7], the Digital Item Adaptation (DIA) part of MPEG-21, and the Descase Ontology ${ }^{6}$. It is remarkable that most industrial standards, such as MPEG7 and MPEG-21, provide detailed descriptions of the user and the user's environment, but using XML Schema to detine their models. Although some initiatives [8] [9] have considered a semantic version of these standards, their results are not publicly available. In the conclusions section we point out a promising solution.

The MIG user device characterization comprises technical details such as display size, color bits pcr pixel, and browser type. Most modern browsers send the "User-Agent"(UA) siring to the server in each HTTP message. MG exploits this feature in order to detect the browser type, the Operating System, and the device's model when a device is used. MIG compares the UA string sent by the user's browser to WURFL ${ }^{7}$, a UA's repository. This repository stores information about 9000 devices, with hundreds of possible capabilities (e.g. is_wireless_device or resolution_width). If the UA is found in the repository, most data concerning the terminal capabilities can be obtained from the repository. The current MIG implementation only considers display size and color bits per pixel.

The MIG user preferences is an arbitrary taxonomy with concepts such as simple, bamque or minimalist. The user profile provides an ordered list of preferred aesthetics.

\subsection{Finding the Best Template}

As pointed before, the model oblained from merging the semantic information from VPOET, MIG, common ontologies and linking elements, can be queried by means of semantic querying languages such as SPARQL. Although the results of a given query depend on the information stored in the model, as we saw in the matching example, and the same query can return 0 or many results depending on key linking elements, what happens whon many results match the query? The only way we have to constrain the results to reach "the best"template for a given user profile is adding more parameters to the SPARQL query.

The problem with using one SPARQL query is that it can return many results, with no sorting criteria, o none at all. The first case denotes a qucry too relaxed, and the second one a query too restrictive.

The solution adopted considers a set of SPARQL queries, ordered from less restrictive to more restrictive, i.e. with fow

6. See http:/diseaseontology-sourceforge net

7. Sec http:/developer.openwave.com/dvl/tools_and_sdk/wurt__and_wall parameters to more parameters, according to an importance criteria. For example, the first query can request malching display size, the sccond request matching display size and browser type, and so on. When the first query is fired, if it returns more than one template, the second onc is fired, following this process until no results are found. The last query with results is considered the "best", and anyone of its resulting termplates is used to render the given scmantic data source.

\section{Related Work}

The personalization topic can be reviewed in [10]. End user preferences applied to Semantic Web Services can be found in [11], where different aspects such as user current context, history (usage and context) or corporate data are consídered in order to create user interfaces ror cellular phones. End user preferences applied to the creation of ontologies is addressed in [12], where the concept of vicwpoint is defined.

Adaptive interfaces [13], characterized by their explicit ability to adapt to the end user is a main topic in HumanCompuler Interaction. An extension of the user model, named behavior-based, is used for personalized web browsing in [14]. where the user profile contains information about browsing goal, interest, expertise and browsing bchavior. However the interface is not personalized in the sense of adaptation to the uscr needs. The approach followed by SADIe [15] considers semantic annotation of CSS to facilitate the transcoding of a given annotated web page to the requirements of impaired users. Our approach do not considers transcoding but template sulection based in the user needs.

Concerning technology, Digital Item Adaptation (DLA) [16] is Part-7 of the MPEG-21 standard. DIA bridges the mismatch beiween rich multimedia content and the usage environment. To this end, descriptions of contents and usage environments are provided in XML. These descriptions are modelled conforming to appropriate XML Schemas by considering four aspects: Terminal Capabilities, Characteristics from User, Network and Natural Environment. Accessibility aspects such as visual impairment are considered in great detail (e.g. "LowVisionsymptoms"is comprised of "LoosOfFineDetail", "LackOfContrast", "LighlSensitjyity", "NeedOfLjght", "CcnterVisionLoss". "PeripherialVisionLoss"), all of these with a numerical value to indicate the impairment degree. This specification has not been ontologized.

\section{Conclusions and Future Work}

Many aspects must be solved in order to achieve the semantic agents specialized in intcracting with end users envisioned in the Semantic Web. This work presents an easy to devclop and extend framework and wcb applications oriented to provide developers with a simple HTTP messages based mechanism to provide a web interface personalized to the user profile for handling semantic data. 
We have merged the ontologies and data from VPOBT and MIG, as well as the appropriated "sameAs"equivalences in order to get a unique model. This model is queried by means of SPARQL queries to obtain matching templates for a given user profile. The way to choose the "best"template is an open issue, in which Semantic Web Rules (SWRL ${ }^{8}$ ) or other non-semantic techniques such as classification algorithms, e.g. Clustering (KNN, K/X Means) or Support vector machines(SVM), can be applied.

Futurc work will deal with templates composition, templatetemplate intcraction, and many technical details such as support for client-side languages such as Action Script (to provide users with rich-interfaces based in Flash) or XHTML. Related works, such as the XML2RDF ${ }^{9}$ tool, could help us to generate ontologies from XML. Schemas (such as MPEG-7 and MPEG21 ) in order to convert parts of the ad hoc ontology used into a more standards based ontology, following the Linked Data initiative.

Visit the cvolution of this framework and its semanticallycnabled applicalions at http://code.google.com/p/fortunata.

\section{Acknowledgment}

This work has been partially funded by the Spanish Ministry of Sciencc and Innovation under the projects HADA (TIN2007-64718), METEORIC (TIN2008-02081) and DEDICON (TIC-4425).

\section{References}

[1] M. d'Aquin, C, Baldassante, L. Gridinoc, S. Angeletou, M. Sabon, and E. Motta, "Characterizing Knowledge on the Semantic Web with Watson," in Proc. EON2007, ISWC/ASWC, voT. 329. CFUR Workshop Proceedings, 2007, pp. 1-10.

[2] T. Finin and L. Ding, "Search engines for semantic web knowledge," in Proc. XTech 2006: Buifding Web 2.0, 2006.

[3] M. Davis, "Semantic Wave 2008 Report: Industry Roadmap to web 3.0 \& Multibillion Dollar Market Opportunities, Execulive Summary." ProjectX10, Tech, Rcp., 2008. Sce www.project10x.coms

[4] T. Bemers-Lee, J. Ifendler, and O. Lassila, "The Semantic Web," Scientific American, vol, 284, no, 5, pp. 28-37, 2001.

[5] M. Rico, D. Camacho, and Oscar Corcho, "VPOET: Using a Distributed Collaborative Platform for Sernantic Web Applications,". Proc. IDC'2008, vol. 162. Springer, SCI Scrics, 2008, pp. 167-176.

[6] M. Rico, D. Camacho, and Oscar Corcho, "A Contribution-based Framework for the Creation of Semantically-enabled Web Applications," submitted for publication to Joumal of Information Sciences, 2009.

[7] S. Karim and A. M. Tjoa, "Towards the use of ontologies for improving user interaction for people with special needs," LNCS, vol. 4061, pp. $77-84,2006$.

[8] J. Hunter, Adling Multimedia to the Semantic Web: Building and Applying an MPFG-7 Ontology, John Wiley \& Sons Ltd, 2005.

[9] R. Troncy, W. Bailer, M. Hansenbias, P. Hofmair, and R. Schlatte, "Fnabling Muttimedia Metadata Internperability by Defining Formal Sernantics of .MPEG-7 Profiles," in Proc. SAMT 200, LNCS vol. 4306, 2006, pp. 4I-55.

[10] J. O. Blom and A. F. Monk, "Theory of Personalization of Appearance: Why Users Personatize their PCs and Mohile Phones," HumanComputet Interaction, wol. 18, no. 3, pp. 193-228, 2003.

8. See http://www.w3.org/Submission/SWRL/

9. Sec http://rhizomik,net/redeter
[11] D. Khushraj and O. Lassila, "Ontological Approach to Gencrating Personalized User Interfaces for Web Services," Proc. ISWC, vol. 3729, pp. $916-927,2005$.

[12] R. Thomopoulos, "Expressing preferences in a viewpoint ontology" Proc. COOPIS, DOA, and ODBASE, PT 2, vol. 3761, pp. 1596-1604, $2 m 05$.

[13] M. Forins, "Graceful Degradation: a Method for Designing Multiplatroorm Graphical Uiser Interfaces," Ph,D. disscrtation, Université catholique de Louvain, 2006.

[14] M. Sah, W. Hall, and D. C. D. Roure, "Designing a Personalized Semantic Web Browser," in Proc. International Conference on Adaptive Iypermedia and Adaptive Web-Based Sy,stems (AH2008), LNCS vol. 5149, pp. 333-336, 2008.

[15] S. Harper and S. Bechhofer, "Sadic: Structural Senantics for Accessibility and Device Independence," ACM Trans, Comput.Hum. Interact., vol. 14, no. 2, p. 10, 2007.

[16] A. Vetro and C. Tïmmerer, "Digital Iten Adaptation: Overview of Standardization and Rescarch Activitics," IEEE Transactions On Melitimedia, vol. 7 , no. 3, pp. $418-426$, Jun. 2005 . 